\title{
A Context-based Mediation Approach to Compose Semantic Web Services
}

\author{
Michael Mrissa, Chirine Ghedira, and Djamal Benslimane \\ Claude Bernard Lyon 1 University, Lyon, France \\ \{firstname.lastname\}@liris.cnrs.fr \\ Zakaria Maamar \\ Zayed University, Dubai, U.A.E \\ zakaria.maamar@zu.ac.ae \\ Florian Rosenberg and Schahram Dustdar \\ Technical University of Vienna, Vienna, Austria \\ \{florian,dustdar\}@infosys.tuwien.ac.at
}

\begin{abstract}
Composition of Web services is a keystone towards the development of interoperable systems. However, despite the widespread adoption of Web services, several obstacles still hinder their smooth automatic reconciliation when being composed. Consistent interpretation of data exchanged between composed Web services is hampered by different and implicit modeling assumptions and representations. In this paper, we look into the value-added of context to enrich data exchange between Web services. We present a context-based mediation approach for semantic Web services engaged in composition.
\end{abstract}




\section{Introduction}

Developed around a platform-independent protocol stack that heavily relines on standards like SOAP [2], WSDL [4] and UDDI [26], Web services ${ }^{1}$ are now widely adopted as a standard means to interconnect applications over the Internet. Web services reach their full potential when composed into business processes that provide users with value-added functionalities. Composition means orchestrating several Web services according to a business process, which is specified with a composition language. Nowadays, WS-BPEL [5] is the de facto standard for Web services composition. Coming from the domain of workflow management, WS-BPEL provides several constructs that permit handling complex interactions between business partners, such as concurrent invocation, fault recovery and conditional switches.

Despite a wide adoption, WS-BPEL still remains focused on syntactical aspects with no emphasis on semantic composition aspects. Moreover, the Web service protocol stack was not initially developed for satisfying the requirements of a successful semantic exchange. Semantic exchange requires Web services to understand the content that interactions permit conveying, i.e., being able to correctly interpret the semantics of the data they send and receive. To meet such requirements, recent work from the Semantic Web community aims at explicitly describing the semantics of Web services. Several languages and frameworks (e.g., WSMO [1], SESMA [11], DIANE [13],

\footnotetext{
${ }^{1} \mathrm{~A}$ Web service is a software component that is described and being accessed via standard XML-based protocols.
} 
OWL-S [15], and WSDL-S [17]) aim at propelling Web services to the level of semantic Web services. Such approaches use ontologies (shared descriptions of a domain knowledge [10]) as agreements on a common vocabulary. Efficient description and management of the semantics of data are major requirements to the success of system interoperability.

However, the aforementioned initiatives do not exploit the potential that context offers when it comes to describing the different aspects of data. The use of context representation has been widely explored in the domain of multidatabase systems [12] in order to clarify the semantic and schematic aspects of data, but is barely studied in the field of Web services. The term context relates to the collection of implicit assumptions that are required to perform a correct interpretation of data. We advocate that accurate data interpretation not only depends on a reference concept, but also on several properties and characteristics that form its context of interpretation. This context needs to be explicitly specified, so related data can be clearly understood. Particularly in the case of Web services composition, meeting the challenges of automatic semantic interpretation and data flow handling requires explicit context description and management.

In this paper we aim at investigating the automated semantic reconciliation of semantic Web services. The rationale of this reconciliation is backed by Maamar et al., who argue that a contextual semantic composition of Web services is subject to satisfying two conditions [14]. The first condition is that Web services must agree on the meaning of the exchanged data, and 
the second condition is that semantic-data conflicts must be automatically resolved using the information that context caters. In this paper, we focus on data heterogeneities that arise when Web services from different origins take part in a composition. We propose to annotate Web services' descriptions with data semantics. This annotation is integrated into a semantic-mediation architecture that handles information heterogeneities using semantic objects and Web services' contexts.

Our main contribution in this paper revolves around a proposal for context-based mediation for reconciling semantic Web services engaged in composition. A mediation architecture is presented, based on first, the contextual annotation of WSDL messages, and second, a model that supports explicit description of context, both validated in previous work $[19,20]$.

The rest of the paper is organized as follows. Section 2 reviews literature on semantics and mediation of Web services. Section 3 presents a motivating example and summarizes the different types of implicit assumptions that can hamper the correct interpretation of data exchanged between Web services. Section 4 summarizes our context model for describing data semantics of Web services, and proposes a solution to manage context information. Section 5 shows how the previous model is integrated into a mediation framework for WS-BPEL business processes, and details the functioning of the different elements of this framework. Section 6 details the prototype developed as a proof-of-concept of our framework. Section 7 concludes the paper and gives some directions for future work. 


\section{Related Work}

This section presents different initiatives related to semantic description and mediation aspects of Web services that helped shape our proposal.

\subsection{Semantic Description of Web services}

At the crossing of the semantic Web and Web services domains, research in the field of semantic Web services is very active. Most approaches intend to describe the semantics of Web services, either with novel semantic description languages $[1,11,13,15]$ or with extensions to syntactic standards $[9,17]$. These approaches bind to domain ontologies in order to explicitly describe the intended meaning of data, including data that Web services exchange.

OWL-S [15] is a subset of the OWL [24] ontology language. It is a general ontology for building semantic Web services, and was designed to be coupled with syntactic description formats like WSDL. OWL-S consists of three elements: service profile, process model, and service grounding, that describe "what the service does", "how the service works", and "how to access the service", respectively. OWL-S advocates to separate the grounding and abstract views when describing the data that Web services exchange. Abstract view binds the data to an OWL conceptual description. Grounding view describes the physical representation of data which generally follows XML Schema [27]. This separation allows different physical representations of the same concept, and strengthens as well the role of ontologies in the abstract 
representation of data semantics.

From the DERI laboratory, WSMF [6] supports the development and description of semantic Web services with a conceptual model. This model recommends maximal decoupling between Web services, and was designed with the idea to enable mediation as a service. WSMO [1] is a formal language and ontology based on the WSMF conceptual model that describes multiple aspects of semantic Web services.

Medjahed and Bouguettaya [16] propose a foundational architecture for semantic Web services. Their work is based on the concept of community, which gathers services from the same domain of interest and publishes the functionalities offered by Web services as generic operations. The authors provide a general template referred to as community ontology for describing semantic Web services and communities. Their work follows a realistic community-centric point-of-view, and adopts a peer-to-peer solution to manage communities, which addresses problems of centralized approaches.

DIANE Elements (DE) and DIANE Service Description (DSD) are objectoriented languages built on a critical analysis of the requirements of semantic Web service description, and on the difficulties of OWL-S and WSMO to fulfill these requirements [13]. DE and DSD use configurable sets and fuzzy logic to support semantic discovery of Web services. DE is a general ontology language with specific features to enhance semantic Web service description. DSD describes services with the constructs provided by DE. DSD revolves around the notions of service request and offer descriptions. 
SESMA [11] is another description format for semantic Web services, that was designed to provide a language with a compact syntax. SESMA supports non-deterministic service description, and is compatible with standards such as WSDL and WS-BPEL. Its main advantage is to be a lightweight language for semantic description of Web services. It remains close to WSDL and WS-BPEL descriptions, and its semantics is not built on top of OWL.

With WSDL-S, Miller et al. annotate WSDL with several extensions related to operations and messages [17]. These extensions refer to concepts of domain models in order to specify semantics of messages, but also preconditions and effects of operations. WSDL-S is also described as a lightweight approach for semantic annotation of Web services.

SAWSDL is a W3C working draft that defines a set of extension attributes for WSDL 2.0 (with WSDL 1.1 support) to describe the semantics of WSDL components [9]. The objective of SAWSDL is to define how semantic annotation of WSDL is accomplished, but it is not intended to specify which language has to be used for the semantic description. It only provides the mechanisms to bind ontology concepts to WSDL annotations.

To wrap up this section, it should be noted that none of the existing approaches use context to describe the semantics of data. Hence, the approach presented in this paper is one step towards enriching Web services' descriptions with context information. Therefore, it should be possible to combine or extend the aforementioned works with the context-based approach we present in this paper. 


\subsection{Mediation between Web services}

Mediation between Web services has received a lot of attention from the research community. Many mediation approaches rely on the concept of mediators for solving data heterogeneities between participants in a composition. Mediators were firstly introduced in the domain of databases [28], and later adapted to the domain of Web services.

Mocan et al. [18] propose WSMX, an implementation of the Web Service Modeling Ontology project (WSMO). WSMX is a mediation architecture for Web services integration. The mediator component is a key part of their architecture and is designed as a service. It mediates concepts between different ontologies that business partners bind to. The mediation solution that Mocan et al. propose relies on a semi-automated graphical interface that allows users to define conversion rules between ontologies. The end-user is helped by suggestions from the system, based on the structure of concepts and already established relations between concepts.

Cabral and Domingue [3] provide a broker-based mediation framework to compose semantic Web services. Their approach follows the WSMF conceptual framework. The mediator component is a key part of their architecture and mediates concepts between ontologies that business partners refer to. Williams et al. [29] use agents to perform semantic mediation between input and output parameters of Web services by encapsulating the composition into an agent, that controls the operation progress.

Spencer et al. [25] present a rule-based approach to semantically match 
Web services' outputs and inputs. A description-logic reasoning system analyzes OWL-S descriptions and generates multiple data transformation rules. This approach focuses on the conversion between different representations of matching OWL-S classes.

While mediation and semantic description of Web services in a composition are very active research fields, to the best of our knowledge, none of these works actually considers context to solve semantic heterogeneities of data in Web services composition. In the following, we present a context-based mediation approach while arguing the benefits of context in the domain of Web services.

\section{Limitations of Semantic Approaches}

In this section, we demonstrate using the classical travel example the need for additional meta-information to accurately interpret the data exchanged between Web services. We stress as well the limitations of current semantic approaches relatively to the concern of semantic interpretation of data.

\subsection{Motivating Example}

We consider a user who plans a trip to Japan, and wishes to rent a car during her stay. The WSDL files of Flight Booking and Car Rental companies are composed with the help of a graphical composition editor ${ }^{2}$ in order to consti-

\footnotetext{
${ }^{2}$ Examples include Oracle BPEL Designer, IBM BPWS4J Editor, Vergil VCAB Composer, or Active Endpoints ActiveWebflow Designer.
} 


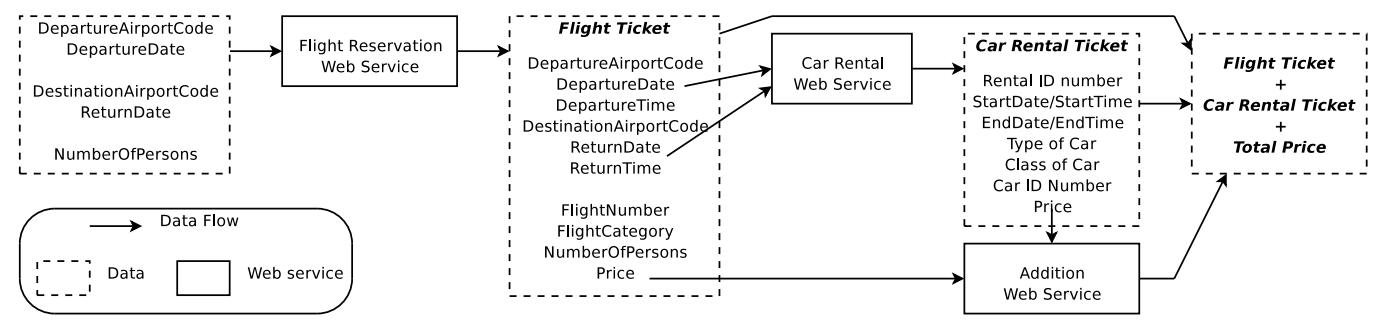

Figure 1: Flight-booking and car-rental Web services

tute the workflow presented in Fig. 1. A basic Addition Web service is used to deliver the sum of flight booking and car rental prices. This Web service is deemed appropriate because of the inherent limitations of XPath, which only supports integer arithmetic and is not intended to perform arbitrary computations [5]. In addition, we assume that the data flows between Web services verify low-level data compatibility. By low-level compatibility, we mean that data objects are described with the same data types (generally with XML Schema type system). This verification is easily performed by the composition editor program.

In Fig. 1 flight-booking Web service computes prices in Euros and with a scale factor ${ }^{3}$ of 1 because the WSDL file was provided by the European branch of the company. However, car-rental Web service uses local currency, i.e. Japanese Yens, and a scale factor of 1000, so there is a need to multiply the value exchanged by 1000 to obtain the actual price. Prices need to be converted into the same currency and scale factor before they can be added and presented to the user.

\footnotetext{
${ }^{3} \mathrm{~A}$ number used as a multiplier in scaling (WordNet, http://wordnet.princeton.edu/).
} 
Moreover, date and time representations differ. Flight-booking Web service uses an European notation (dd.mm.yyyy and 12:00 AM/PM), whereas car-rental Web service uses a Japanese notation (yy.mm.dd and 24:00). This example, albeit simple, shows so far how much low-level compatibility is insufficient to meet for the requirements of semantic exchange. Indeed the interpretation of data will be inaccurate because data bind to different contexts and should be interpreted differently.

The WS-BPEL community currently solves semantic heterogeneities by manually describing the conversion of data between different semantic representations. However, this solution is tedious and not scalable. Plus it needs to be performed upfront (i.e., at design time) by domain experts, and relies on XSLT stylesheets and XPath expressions to convert data. It requires experts to have technical knowledge about both XPath and XSLT, and about the semantics of the domain concerned. Last but not least, it is not adapted to on-the-fly associations between Web services.

In the following, we discuss the main types of heterogeneities related to the implicit assumptions that can hamper a correct interpretation of domain ontology concepts

\subsection{Heterogeneous Implicit Assumptions}

The role of domain ontologies is to provide users with an agreement on the interpretation of described concepts. Therefore, the different semantic properties related to domain concepts remain implicit in domain ontologies. With 
semantic properties, we refer to the different aspects and characteristics that permit to establish the context of the current interpretation of a concept. These assumptions on the interpretation of concepts remain implicit in the ontology, because the initial purpose of ontologies is to describe a domain knowledge, which includes concepts and the relations between them, and also relevant properties of concepts, but not their different possible contexts of interpretation.

From a provider's point of view, performing semantic interoperability with ontologies is often tedious, because Web services have usually different interpretations of the same concept. Hence, providers need to adapt their own implicit assumptions on the interpretation of concepts to the assumptions of ontologies. Solving data interpretation discrepancies remains at the charge of Web services providers. This task is still performed manually and at design time. Discrepancies between semantic properties are due to different implicit assumptions on data interpretation. These discrepancies are summarized as follows.

\subsubsection{Value Heterogeneities}

Domain ontologies often make implicit assumptions about the values of semantic properties. As shown in Section 3.1, prices are generally assumed to have a scale factor of 1 . However, some organizations usually handle prices with a scale factor of 1000 . In this case, we say that the values of the scale factor semantic property differ. Resolving such heterogeneities requires ex- 
plicit description of the semantic property and its associated value.

\subsubsection{Structural Heterogeneities}

Domain ontologies implicitly follow a static structural representation of semantic properties that are relevant to the described application domain. However, it appears that even in the same domain, different semantic properties and structural organizations could be relevant to Web services providers.

For example, price concept could have different relevant semantic properties depending on the needs of travel agencies. Some agencies could use different scale factors and ignore the currency aspect because their partners all use the same currency, whereas some other agencies could make opposite assumptions. These structural heterogeneities between semantic properties need to be explicitly described, so it becomes possible to determine if a particular structure of interpretation of a concept is compatible with another.

\subsubsection{Semantic Heterogeneities}

Semantic heterogeneities relate to semantics used for describing semantic properties. They are not visible as long as the context of interpretation remains implicit, but appear when the context is explicitly described. For example, an English provider could use the word "VATIncluded", whereas a French one would use the word "TVAIncluse" to describe the same semantic property, which specifies whether or not Value-Added Taxes are included in a

price (synonymy conflict). In order to solve such semantic conflicts, explicit 
vocabulary is required to describe semantic properties.

It appears from the above that different types of heterogeneities affect semantic properties. In the following section, we show how the notions of context and semantic object can be used in order to explicitly describe semantic properties. We present afterwards a context-based approach to handle the aforementioned limitations.

\section{A Context Model for Web Services}

In order to overcome the limitations we report in Section 3, we proposed in [19] a context model that is built around the notion of semantic object and context. This model gives providers the means to explicitly describe the implicit assumptions they make on data. In this section, we present the arguments that back this model, and provide insights on the description of context information, before discussing its integration into the Web services model.

\subsection{Context Model: Main Concepts}

The context model revolves around the notion of semantic object. This one extends a data object with additional meta-data, so the context of interpretation of a data object is made explicit. Basically, a semantic object contains (1) a data part that has a value $v$ of type $t$ described in a type system language, and (2) a semantic part that has a concept $c$ of the application 
domain, and a context $C$ represented as a tree of meta-attributes, referred to as modifiers. Modifiers make explicit the semantic properties of semantic objects. They are also semantic objects, so they have a value, a type, a domain concept they refer to and possibly a context. Web services providers need to know these modifiers for a correct interpretation of a semantic object. Semantic objects with different contexts may be converted into a common context, which allows to compare them and even to enable data exchange between Web services.

For the needs of our model, we defined two categories of modifiers: static and dynamic. It is mandatory to provide static modifiers in order to make clear the semantics of a semantic object. On the contrary, dynamic modifiers can be inferred from other modifiers belonging to the same semantic object. More details about the properties of modifiers and the possibilities of conversion raised by this model are given in [19]. In order to illustrate the proposed context, let us consider the following example:

- concept = domain_ns:price,

- value $=5$,

- type = xsd:double,

- Context $=[$

- (ctxt_ns:currency, xsd:string, "euro", [

* (ctxt_ns:country, xsd:string, "France", null)

* (ctxt_ns:date, ns:date, 15.05.2005, [

• ctxt_ns:dateformat, xsd:string, "dd.mm.yyyy", null ]]

- ctxt_ns:scalefactor, int, 1, null 
- ctxt_ns:VATIncluded, xsd:bool, true, [ * ctxt_ns:VATRate, float, 19,6, null ]

- ] //end of context

This example describes price as a semantic object along with its contextual characteristics. To handle a concrete price, one needs to know the following details: currency, date related to this price due to currency changes over time, and Value Added Taxes (VAT) rate applied to this price. We notice from this representation that (ctxt_ns:country, xsd:string, 'France"', null) modifier is static, i.e, it cannot be inferred from the values of other modifiers. This static modifier can help infer the "euro" value of the currency modifier, being given a rule that states Euro is the currency of France. Currency modifier is then qualified as dynamic. In addition, we notice that country modifier helps infer the value of dateformat, which is then a dynamic modifier. Accurate interpretation of the price semantic object is then possible with the attached context.

The multiple cases of heterogeneity presented in Section 3.2 can now be explicitly handled with context, and thus will not be viewed as implicit discrepancies anymore, but rather as well-known heterogeneities between instances of schemas. Indeed, context information could be directly added to the domain ontology. However, we explain in the next section why we deem appropriate to separate context from domain knowledge, and to store context information into dedicated context ontologies. 


\subsection{Context Representation and Integration}

In this section, we detail our recommendations on how to store the information required to represent context. To this purpose, we first give an insight on the different strategies for ontology design. Then, we present the advantages of context ontologies, and how they are separated from domain ontologies, before presenting our WSDL annotation that achieves the integration of context into the Web services protocol-stack.

\subsubsection{Strategies for Ontology Design}

The design of a domain ontology can be seen from different perspectives, which are referred to as top-down, bottom-up and middle-out approaches [22, 23]. A top-down approach consists in first agreeing on the most general concepts in order to provide a shared representation of the world. This shared representation is then adjusted for the sake of meeting the needs of the local views of the participants. It is a very reliable strategy in a limited environment, in which the number of participants and the discrepancies between their views of the world, are limited. However, it is not efficient in an open world like the Internet. Indeed, it is not guaranteed that an unknown and constant changing number of participants could agree on a single view of the world. On the contrary, a bottom-up approach starts from the local and very specific conceptualizations of the participants, and follows a generalization process towards a consistent representation of the domain knowledge. Compared to the top-down approach, this approach is more adapted to in- 
teractions in an open world, but it appears to be less efficient in a limited environment.

The middle-out approach strengthens the middle concepts of the ontology into identifiable groups, and follows both specialization and generalization steps to build the domain knowledge representation. Generally, it is recommended to combine top-down, bottom-up, and middle-out approaches, in order to model the domain knowledge of different participants in an ontology [23]. In our case, we identify two of these three approaches and associate them with context and domain ontologies, in order to describe providers' semantics.

\subsubsection{Context vs. Domain Ontologies}

Our proposal to make the distinction between context and domain knowledge comes from the fact that the heterogeneities presented in Section 3 do not concern the domain knowledge itself, but rather relate to providers' local and yet implicit assumptions on the interpretation of domain concepts. These assumptions, referred to as context, are related to cultural, geographical, and temporal situations of Web services, e.g., when, where, and how they are designed, deployed, and executed.

In most cases, when several participants intend to agree on a shared domain ontology, they already have different contexts. Especially in an open world like the Internet, we have seen that it is very difficult to reach a common agreement on a shared representation of a domain knowledge when adopting a 
top-down approach only. This is mainly due to the different, already-existing contexts of the participants. As a consequence, we set several objectives to facilitate both ontology design and the reconciliation of Web services:

- focus the role of domain ontologies on describing knowledge that can be agreed on with a top-down approach;

- adopt a bottom-up approach to reconcile the contexts of Web services, as they already exist before adhering to a domain ontology;

- and, give providers the responsibility of describing the structural and organizational aspects of their local contexts, and of providing the connections to other providers' contexts when they adhere to a domain ontology.

In order to meet these objectives, we define the notion of context ontologies, which are intended to make context explicit for each concept in a domain ontology. The difficulties raised when agreeing on a shared representation of a domain knowledge remain, but they are now identified and isolated, due to explicit and separate description of context heterogeneities. Thus, context heterogeneities can be handled, because contexts of participants are explicitly described in context ontologies. Rich description languages like OWL allow specifying such relations between ontology concepts. The separation between context and domain ontologies is illustrated in Fig. 2, in order to clarify the terminology used for context description. 


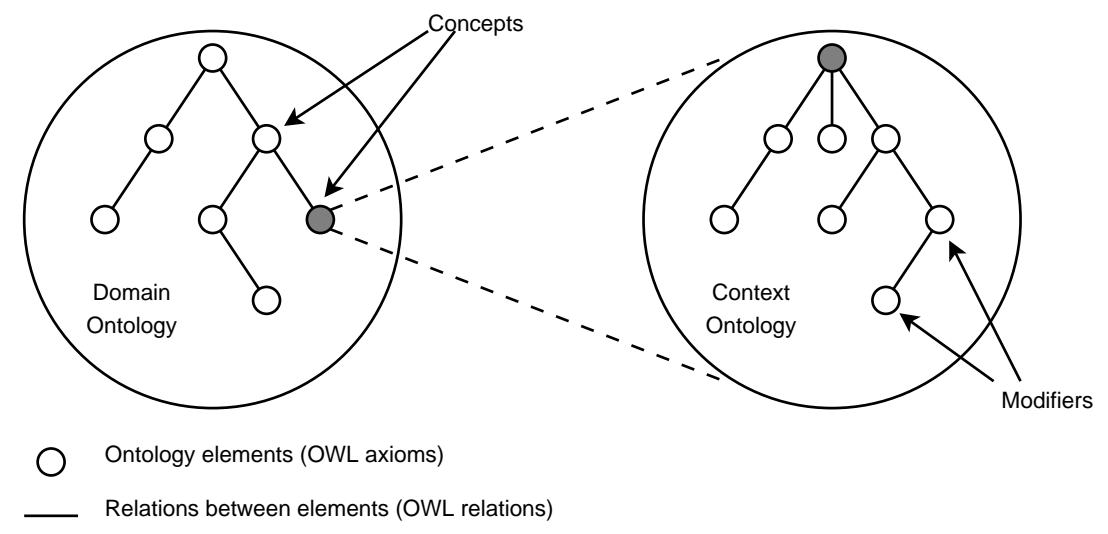

Figure 2: Context vs. domain ontologies

As context ontologies provide shared vocabularies to specify structural and semantic representations of context, there is a need to extensionally specify the values that modifiers take. We recall that modifiers are either static or dynamic. Consequently, we develop two different solutions to instantiate modifiers. We insert a description of static modifiers into WSDL in a way to remain compliant with the Web services protocol-stack [19]. Descriptions of static modifiers provide the means to calculate dynamic modifiers at runtime, using appropriate inference rules.

The use of context ontologies and WSDL annotations helps providers make explicit the context of data. It provides a scalable solution to integrate context into the Web services protocol-stack. Moreover, it enables semantic mediation of data during the execution of a composition. In the following, we give an overview of our solution, detailed in previous work [20], for annotating descriptions of composed Web services. 


\subsection{Extending WSDL with Context}

Using the model of Section 4.1 requires enriching the description of Web services with context, by annotating WSDL message parts, so they can be now considered as semantic objects. In WSDL descriptions, <message> elements describe data exchanged for an operation. Each message consists of one or more <part> elements. We also refer to <part> elements as "parameters" in the rest of this paper. Each parameter has <name> and <type> attributes, and allows additional attributes. Our annotation takes advantage of the extension proposed in the WSDL specification [4], so that annotated WSDL operates seamlessly with classical and annotation-aware clients. To keep the paper self-contained, we overview a simplified structure of the annotated WSDL meta-model in Fig. 3.

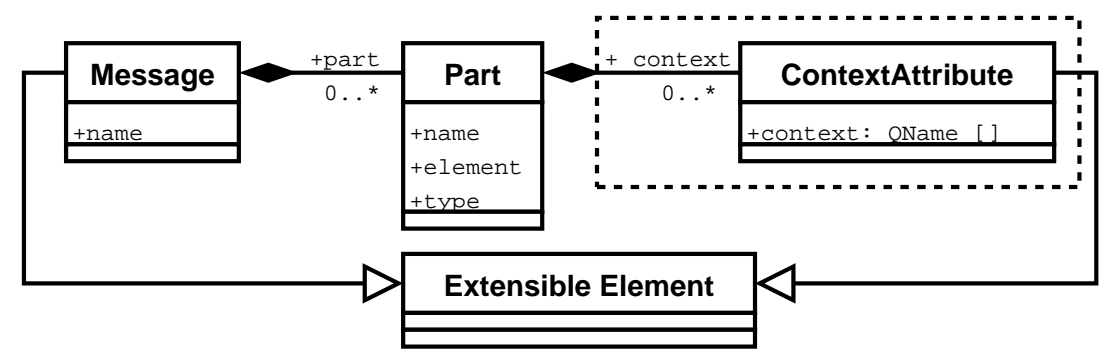

Figure 3: Partial representation of the extended WSDL meta-model

<part> elements are annotated with a context attribute that describes the names and values of static modifiers using a list of qualified names. The first qualified name of the list specifies the domain ontology concept of the value $(c)$. Additional elements refer to instances of static modifiers described in a context ontology. Listing 1 illustrates the proposed extension with Car- 
Rental Web service of Section 3.1. The annotation shows the values taken by static modifiers in this Web service.

Relying on this annotation, a value $v$ and its data type $t$ described in WSDL are enhanced with the concept $c$ and the necessary modifiers to define the context $C$, thus forming a semantic object $\langle c, v, t, C\rangle$. To complete the context $C$, rules help infer the values of dynamic modifiers at runtime. This offers several advantages: rules are easily modifiable, making this solution adaptable to changes in the underlying semantics. In addition, oftenchanging values of modifiers could not be statically stored, so using rules simplifies the annotation to WSDL. Furthermore, rules separate application logic from the rest of the system, so updating rules does not require rewriting application code. In the following, we detail our context mediation architecture that integrates mediators into composition as Web services, and show its internal functioning, which relies on rule-based mechanisms. Our mediation architecture aims at reconciling Web services at the semantic level.

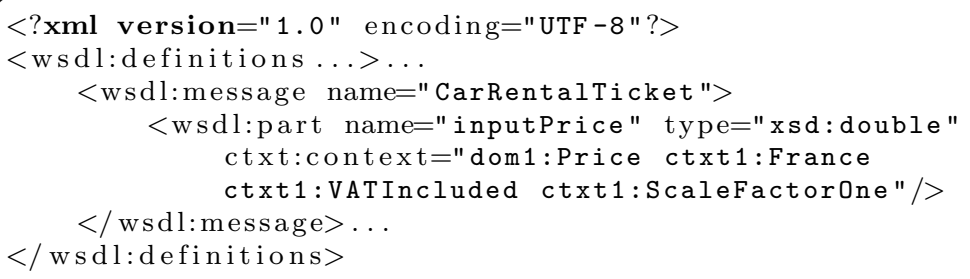

Listing 1: Car Rental Annotation Snippet 


\section{A Context-based Mediation Architecture}

In this section, we present a context-based mediation architecture that takes advantage of the features offered with the aforementioned context model. Firstly, we discuss the advantages of service-based integration of mediators into the composition. Secondly, we give an overview of the proposed architecture with a WS-BPEL business process based on the example of Section 3.1, before detailing the generation of contextualized business processes that include mediator Web services.

\subsection{Advantages of Service-based Mediation}

The solution proposed in this paper follows a service-based approach to implement mediators, referred to in the following as mediator Web services. Service-based mediation presents several advantages. First, the standardized access to Web services through their WSDL descriptions allows better independence from composition languages and engines. Second, managing the mediation concerns in a service-oriented way is more scalable, because it does not require extending any language, neither modifying existing composition architectures, but rather reusing existing deployed software components. Third, the loosely coupled aspect of a service-based architecture allows keeping mediation concerns independent from original functionalities of Web services.

However, the main issue is the need to adapt input and output data 


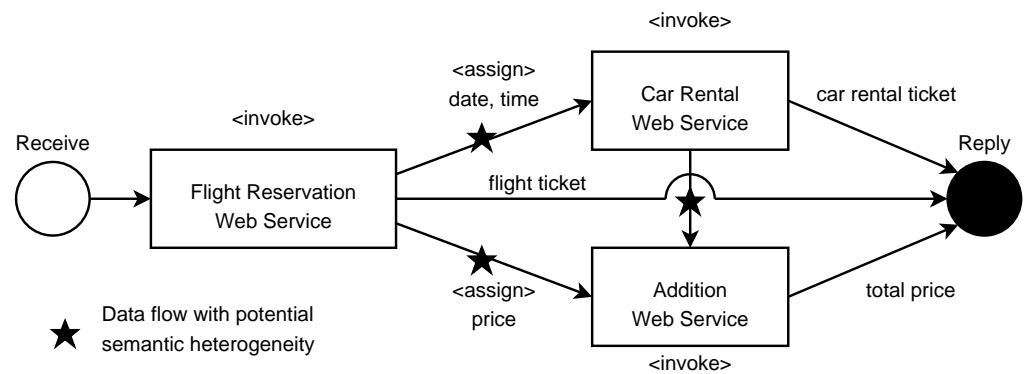

Figure 4: View of the original business process

of mediator Web services to the data representation expected by the Web services they communicate with. We answer this limitation in the following and propose a solution that can be applied as a pre-deployment step, during the deployment of a composition.

\subsection{Overview of the Mediation Process}

For consistency purposes, we continue using the example of Section 3.1 in order to illustrate the mediation process. The BPEL process depicted in Fig. 4 implements the composition logic of the workflow shown in Fig. 1. It has been previously demonstrated that several heterogeneities hamper the correct execution of this workflow. We assume that the WSDL files used in this paper are correctly annotated with context information [20], and that the corresponding domain and context ontologies are available. Our mediation approach is a three-step process, as shown in Fig. 5.

Contextualization step. A contextualization algorithm (Section 5.3) analyzes the BPEL process (i.e., Fig. 4) to locate explicit and implicit data 
flows. Relevant data flows for possible context heterogeneities are symbolized with stars. In our running example, the data flows of interest are: a) where the price variables are sent to the addition Web service, and b) where the date and time variables are sent to the Car-Rental Web service.

Automatic mediator Web services generation step. Here a mediator Web service is automatically generated and deployed for each data flow where the contextualization algorithm detected possible context heterogeneities. The generation of mediator Web services is handled by our Web Service Code Generator (WS-CG), to be described in Section 6.1. Each generated mediator Web service implements an operation, named mediateX2Y, where $X$ and $Y$ are replaced with the names of the input and output messages of operations specified in the WSDL files related to the mediator Web service. Inputs to the mediation operation are the values, which have to be transformed into the representation specified in context annotations. Outputs of the operation are the transformed values that have the expected meaning for the target operation in the composition. Details on the runtime generation and deployment of mediator Web services are given in Section 6.1.

Updating original composition process step. Invocations to the generated mediator Web services from step 2 are inserted into the original BPEL code according to Algorithm 1. The new BPEL code uses the endpoints of dynamically generated mediator Web services combined with required <invoke> elements. A general template for mediator Web service invocation is depicted in Listing 2. After replacing all implicit and explicit 


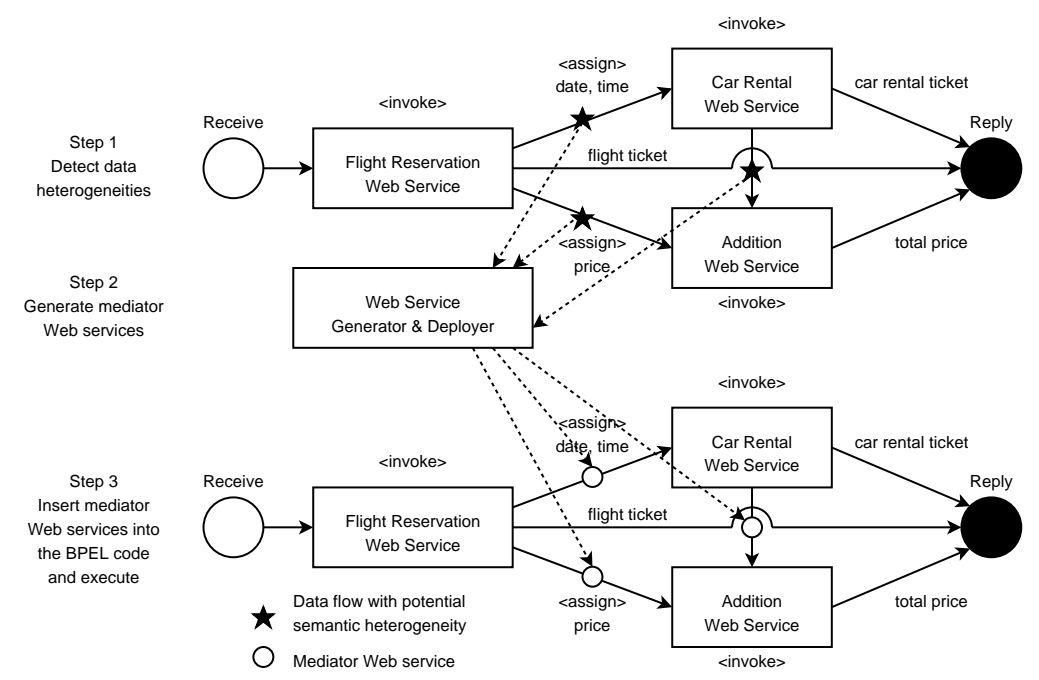

Figure 5: Generation of the mediator Web service

data flows, the contextualized BPEL process is ready to be executed by any usual BPEL execution engine. During the execution of the contextualized BPEL process, mediator Web services are invoked to handle semantic heterogeneities of data. The different steps performed by mediator Web services are summarized in Section 6.2.

\subsection{Dynamic Generation of Contextualized Processes}

WS-BPEL does not always make the data flows in a composition explicit, since it is meant to be "programming at large". Data flows in WS-BPEL are encapsulated in $\langle$ variable $\rangle$ elements. We distinguish between implicitly shared data between partners or explicitly shared data copied using <assign> elements. Our approach consists in locating implicit and explicit data flows and replacing them with invocations to mediator Web services. 
First, let us consider explicit data flows described with <assign> elements. Such elements contain one or more <copy> elements, themselves containing a $<$ from $>$ element and a $<$ to $>$ element, that respectively describe where data comes from and where it goes. Mediation is concerned with elements that are assigned from a variable to another variable only. We assume that the semantics of data entered manually by the composition designer (expressions or literal values) matches the semantic requirements of the business process. To integrate mediator Web services into WS-BPEL, we replace selected <assign> statements with an invocation to a generated mediator Web service, with the sequence depicted in Listing 2.

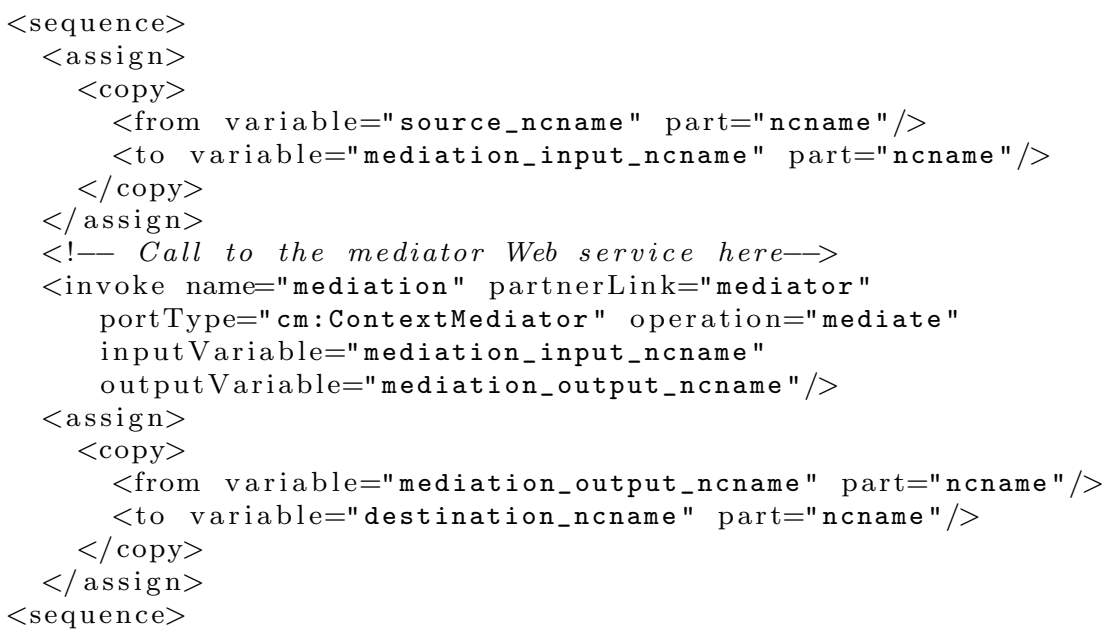

Listing 2: Mediator Invocation in BPEL

<sequence $>$ element allows consecutive execution of the contained elements by firstly, building the input message for the mediator invocation by using <assign> activity. Secondly, the mediator is invoked using <invoke> element followed by <assign> activity to extract the mediated data from 
the output message to the destination variable. By replacing the original <assign> element with the BPEL code presented in Listing 2, the mediator Web service is inserted into the BPEL composition to intercept and adapt the data flow. The generation of the mediator Web services from the WSDL descriptions of the source and target Web services, is described in detail in Section 6.1.

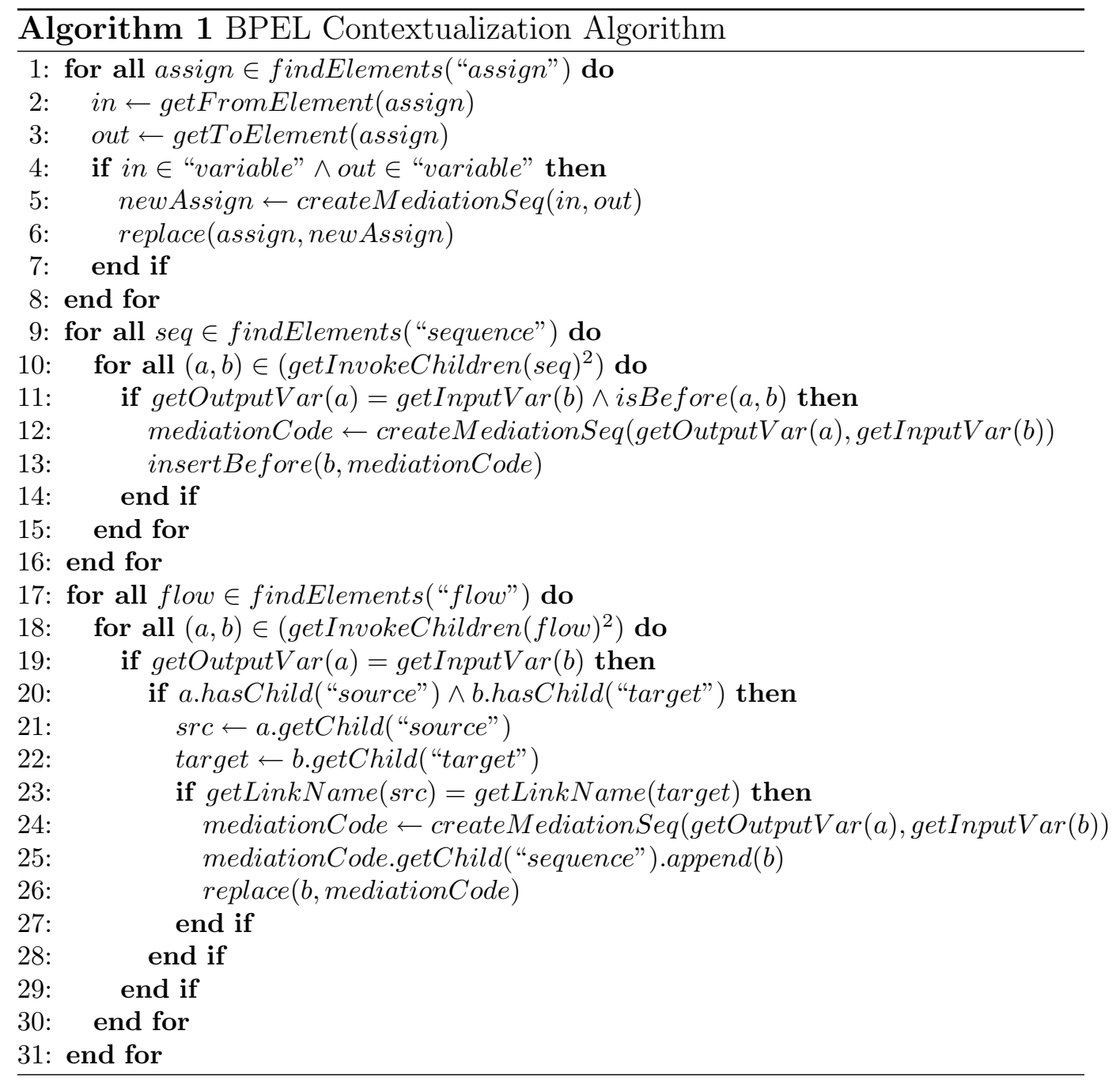


To handle implicit data flows, we need to locate shared variables, i.e., variables that are first used as output of <invoke> element, and then directly used as input of another consecutively executed <invoke> element. In BPEL this situation happens in the following cases: (1) <sequence> element contains several <invoke> child elements, and $(2)<$ flow $>$ element contains several <invoke> child elements that are bound together through <link> element. Algorithm 1 shows the detection in BPEL of explicit and implicit data flows described previously, and the modifications performed to insert the mediation code described in Listing 2 .

The first part of the algorithm (lines 1 to 8) detects explicit data flows described with <assign> elements. findElements function is used to locate all the assign elements. Then, $<$ from $>$ and $<$ to $>$ child elements are extracted from each <assign> element (lines 2-3) and if both are variables (line 4) they are used by createMediationSeq function to create the mediation code (line 5) that replaces the former <assign> element (line 6).

Lines 9 to 16 show the detection of consecutive <invoke> elements in a sequence. Function getInvokeChildren(sequence) gets the <invoke> child elements that belong to the same sequence. getInputVar(invoke) and getOutputVar(invoke) functions extract the information contained in the attributes called inputVariable and outputVariable of the selected <invoke> element. Function isBefore $(a, b)$ verifies that element $a$ is executed before element $b$ in the BPEL code. So, if two <invoke> elements of a sequence (line 9-10) have matching output and input variables and are in the 
right execution order (line 11), the mediation code (line 12) is inserted just before the second <invoke> operation with insertBefore function (line 13), so that mediation is only performed if necessary. It should be noted that other elements such as <switch> may change the execution of the workflow.

Lines 17 to 31 show the detection of related <invoke> elements in a flow. The algorithm identifies <invoke> elements that have identical inputVariable and outputVariable attributes, which possibly characterizes an implicit data flow (lines 17-19). These elements have to be related to each other by containing a <source $>$ and <target $>$ children that have the same linkName attribute (lines 20-23). In this case, the generated mediation code (line 24) includes the second <invoke> element (line 25) that is added with append function. So, it replaces the original <invoke> element with a sequence including both the mediation code and the original invocation.

The algorithm described above is essential to generate the contextualized BPEL, which weaves the mediation concern into the original business process, by including calls to mediator Web services generated on-the-fly.

\section{Implementation Work}

A prototype has been developed as a proof-of-concept of the feasibility of this architecture under the Java ${ }^{\mathrm{TM}}$ environment. It includes several components. A graphical user interface enables providers to annotate WSDL files with context. A model-driven Web service generator deploys mediator Web 


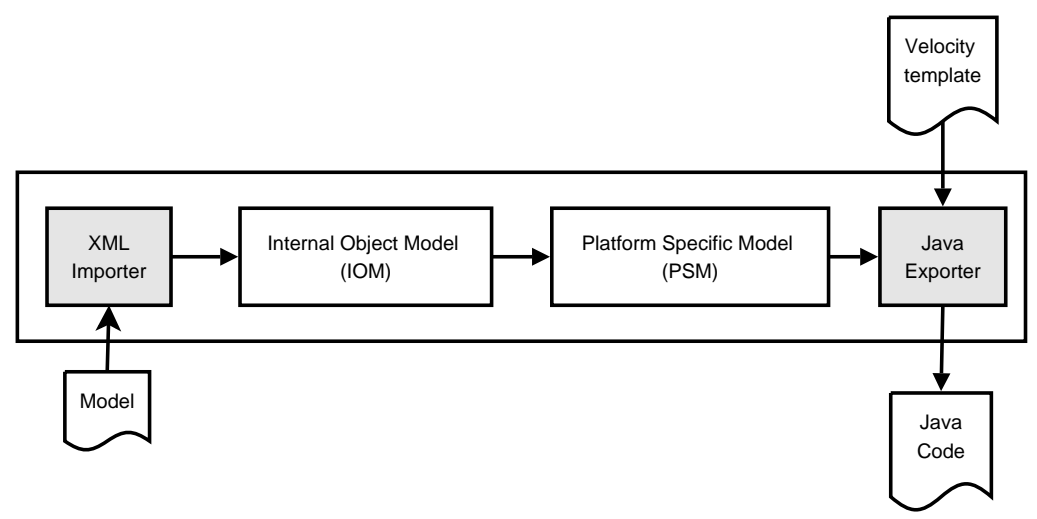

Figure 6: Model-driven Web service generator

services in Axis2 runtime. A mediator Web service has been implemented, which reads in context annotation from WSDL files and converts data from a source context to a target context. The contextualization algorithm for WS-BPEL processes has also been implemented.

\subsection{Model-Driven Web Service Generation}

During the contextualization process of the original BPEL process, one or several mediator Web services need to be generated. Therefore, we implemented a flexible and lightweight model-driven code generator based on a platform independent object model (IOM). Based on the IOM, we implemented a platform specific model (PSM) for the Java platform.

The main elements of the code generator are shown in Fig. 6. A model description specified in XML acts as an input to the code generator. The input file is parsed by an XMLImporter component, which builds the IOM corresponding to the XML description of the model. The IOM is then transformed 
into a PSM oriented Java. JavaExporter component operates directly on the PSM and iterates through every class, interface, etc. to generate the Java code. The actual code generation is supported by the Apache Velocity template engine [8]. Based on this model-driven code generator, we implemented a special Axis2CodeGenerator component for generating a Web service for the new Axis2 [7] runtime, which executes the following steps:

1. Dynamic code generation and compilation: the aforementioned code generator is used to dynamically generate and compile a Java class, which will be deployed as Web service. All libraries for compiling the code are dynamically added to the classpath.

2. Deployment descriptor generation: Axis2 requires a special deployment descriptor called services.xml, which specifies the main class implementing the service logic. Additionally, each operation of the class which should be exposed as Web service operation has to be specified. Axis2 implements purely document-style Web services by leveraging a top-down (or "WSDL-first") approach. Due to the fact that we generate the implementation of the Web service directly, we use a bottom-up approach, which is typically used for RPC-style Web services. Therefore, we use a special message handler, called RPCMessageReceiver, which is specified in the deployment descriptor. It is responsible for converting the documentstyle nature of a Web service as required by Axis2 to the RPC structure we use internally (by exposing a Java class as Web service). 
3. Code packaging and deployment: the compiled code together with the deployment descriptor and the required libraries are packaged together into an .aar file (Axis2 archive). The new deployment model of Axis2 allows a very simple deployment step. The archive file created in the previous step is simply copied to the Axis2 deployment directory (specified via properties in our system). The deployment itself is then handled by the Axis2 runtime.

On the basis of the Axis2CodeGenerator, we developed a component named MediationServiceBuilder that currently builds the mediator Web service based on the WSDL files and the operation names it gets as an input. These input data define the services and the data types for which a mediator has to be generated. Based on these input data it dynamically builds a model for the Web service, consisting of a Java class and one operation. The implementation of the mediator operation is presented in detail in the next section.

One important aspect about the life cycle of the mediator Web service is the fact that it automatically gets undeployed if the corresponding BPEL process is undeployed. At a technical level this is achieved by registering a deployment listener to the BPEL engine to receive notifications about the deployment or undeployment of various processes. If the MediationServiceBuilder receives such a notification it has to determine the corresponding mediator Web service and undeploy it from the Axis2 runtime. 


\subsection{Operation of Mediator Web Services}

In this section, we detail the internal operation of mediator Web services. The latter are inserted into composition processes between original Web services that may have context heterogeneities. We discuss the mediation steps performed by mediator Web services with the example of this paper. We consider the data flow from Car-Rental Web service to Addition Web service, passing through the mediator Web service. This one takes as input "price" message part sent by Car-Rental Web service, and then performs the steps described in Fig. 7 before sending the result of the computation in a "price" message part, to Addition Web service.

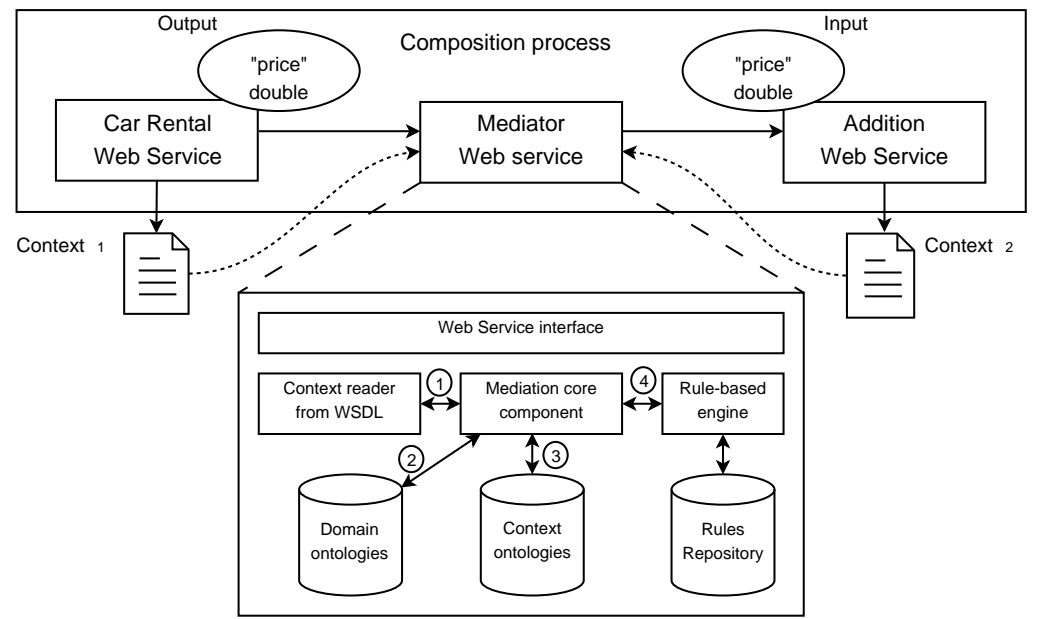

Figure 7: Detailed View of the Mediator Web service

In step one, WSDL files of Car Rental and Addition Web services are fetched by the mediator Web service. Then, these files are parsed in order to extract the required elements. In particular, we are interested in the 
annotations of the messages parts that the mediator receives and sends, in our running example, output and input price message parts of Car-Rental and Addition Web services respectively. Extracted annotations refer to the domain concept and the semantic properties necessary to a correct data interpretation. An example of such an annotation of Car-Rental Web service was depicted in Listing 1.

In step two, the mediator Web service identifies the exchanged concepts in domain ontologies. The annotation is a list of attributes, and the first annotated attribute always refers to the domain concept. Here, the annotated message part refers to price concept of the domain ontology identified with the namespace dom 1 in the WSDL file. The mediator Web service checks that the concepts of both Car Rental and Addition Web services match, i.e., that they verify a subsumption or equivalence relation. This is a simple approach to semantic matching but additional capacities can be integrated into the mediator. For a good survey on semantic integration techniques, see Noy's work [21].

In step three, an in-memory tree is built from the context ontology to represent the context related to price concept. The annotated context attributes corresponding to the price concept are identified and their values are added to the tree. Annotated context attributes refer to OWL individuals (instances), so they describe not only modifiers, but also the values they take in the context of the Web service. In Listing 1, ctxt1:France attribute is an instance of country concept in the context ontology. Moreover 
ctxt1:ScaleFactorOne attribute is an instance of scaleFactor concept in the context ontology. We assume that Web services providers correctly add this information to the context ontology before annotating WSDL files.

In step four, the mediator Web service communicates with a rule engine to perform several tasks. The first task consists in inferring the values of dynamic modifiers that are part of the context, using rules stored in the knowledge base. For example, being given (country $=$ France) as a fact, the rule engine infers $($ currency $=$ euro) by querying the knowledge base. Then, currency modifier is being affected the value euro.

The second task consists in converting the received data into the required context representation. From the previous steps, we obtained two in-memory context representation trees that have valued or not-valued elements. Next, the mediator compares each element of the context and queries the rule engine to see if the values are convertible. If a value is missing, the mediator queries the knowledge base for a rule that specifies a default value. If no such rule exists, the conversion is canceled and an error is thrown. The knowledge base also contains conversion rules that allow dynamic conversion between context values. For instance, price in our example is converted into the required currency by calling a remote component that provides up-todate currency conversion rate. Such conversion needs to be dynamic, so that it can answer the requirements of the temporal perspective of context. 


\subsection{Test Case and Evaluation}

A test experiment has been conducted on the basis of the example developed in this paper. Our current composition example is hosted by an Apache Tomcat container, our mediator Web service implementation uses Jena 2 API and Drools rule engine to access and manipulate OWL ontologies and perform data conversion ${ }^{4}$. The prototype includes illustrative domain and context ontologies for describing the required concepts and contexts ${ }^{5}$.

The implementation performs at-runtime context mediation, enabling meaningful execution of composition. In the example of this paper, not only price concepts match, but data is transformed at-runtime, to comply with the different scale factors, heterogeneous date formats (that allow getting upto-date conversion rates between currencies), and different VAT rates (that also are not always included in the price), described in the context ontology.

As future work, we envision further practical tests and performance evaluation. However such experiments require additional domain and context ontologies that must be validated by domain experts, as well as additional sets of conversion rules and functions. Therefore, and for the purpose of this paper, we limited our experiments to the test-case developed previously, as a proof-of-concept of the feasibility of our architecture. Ongoing work also concerns the integration of the contextualization algorithm with WS-BPEL implementations such as ActiveBPEL ${ }^{\mathrm{TM}}$ or Apache Ode ${ }^{\mathrm{TM}}$.

\footnotetext{
${ }^{4}$ http://tomcat.apache.org/, http://jena.sourceforge.net/ and http://www.drools.org/.

${ }^{5}$ Available at http://www710.univ-lyon1.fr/ mmrissa/
} 


\section{Conclusion}

In this paper, we presented a context-based approach for semantic Web services composition. The approach revolves around the following aspects: annotating WSDL descriptions so that Web services are now described with contextual details, deploying a context-based mediation architecture so that implicit assumptions on data flow are made explicit, and automatically generating and invoking Web services mediators so that data heterogeneities between Web services are handled during the composition.

Future work aims at looking into the following issues. First, unexpected changes in some Web services' non-functional properties could lead to substitute some Web services with others offering the same functionality. The challenge is to make this substitution automatic, dynamic, and transparent. Second, Web services discovery and selection steps could ease semantic mediation during the composition step. The challenge is to ensure that semantic mediation is taken into account during discovery and selection stages.

\section{References}

[1] S. Arroyo and M. Stollberg. WSMO Primer. WSMO Deliverable D3.1, DERI Working Draft. Technical report, WSMO, 2004. http://www.wsmo.org/2004/d3/d3.1/.

[2] D. Box, D. Ehnebuske, G. Kakivaya, A. Layman, N. Mendelsohn, H. F. Nielsen, S. Thatte, and D. Winer. Simple object access protocol (SOAP) 1.1. Technical report, The World Wide Web Consortium (W3C), 2000.

[3] L. Cabral and J. Domingue. Mediation of semantic web services in irs-iii. In 1st Int'l Workshop on Mediation in Semantic Web Services 
(MEDIATE 2005) at the 3rd Int'l Conf. on Service Oriented Computing (ICSOC 2005), Amsterdam, The Netherlands., December 12th 2005.

[4] E. Christensen, F. Curbera, G. Meredith, and S. Weerawarana. Web Services Description Language (WSDL) 1.1, W3C Note. Technical report, The World Wide Web Consortium (W3C), March 2001.

[5] F. Curbera, Y. Goland, and J. K. et al. Business Process Execution Language for Web Services (BPEL4WS) Version 1.1, May 2003.

[6] D. Fensel and C. Bussler. The Web Service Modeling Framework WSMF. Technical report, Vrije Universiteit Amsterdam, 2002.

[7] A. S. Foundation. Axis2. http://ws.apache.org/axis2/ (last accessed: May, 29 2006).

[8] A. S. Foundation. Velocity - Java-based template engine. http:// jakarta.apache.org/velocity/ (last accessed: May, 29 2006).

[9] S. W. Group. Semantic Annotations for WSDL, W3C Working Draft. Technical report, The World Wide Web Consortium (W3C), Sept. 2006.

[10] T. Gruber. What is an ontology? http://wwwksl.stanford.edu/kst/what-is-an-ontology.html, 2000.

[11] Joachim Peer. Semantic Service Markup with SESMA. In Web Service Semantics Workshop (WSS'05) at the Fourteenth International World Wide Web Conference (WWW'05), 2005.

[12] V. Kashyap and A. P. Sheth. Semantic and schematic similarities between database objects: A context-based approach. VLDB J., 5(4):276304, 1996.

[13] M. Klein, B. König-Ries, and M. Müssig. What is needed for semantic service descriptions - a proposal for suitable language constructs. International Journal on Web and Grid Services (IJWGS), 1(3/4):328-364, 2005.

[14] Z. Maamar, D. Benslimane, and N. C. Narendra. What can context do for web services? Commun. ACM, 49(12):98-103, 2006.

[15] D. L. Martin, M. Paolucci, S. A. McIlraith, M. H. Burstein, D. V. McDermott, D. L. McGuinness, B. Parsia, T. R. Payne, M. Sabou, M. Solanki, N. Srinivasan, and K. P. Sycara. Bringing Semantics to Web Services: The OWL-S Approach. In J. Cardoso and A. P. Sheth, editors, SWSWPC, volume 3387 of Lecture Notes in Computer Science, pages 26-42. Springer, 2004.

[16] B. Medjahed and A. Bouguettaya. A dynamic foundational architecture for semantic web services. Distributed and Parallel Databases, 17(2):179206, 2005. 
[17] J. Miller, K. Verma, P. Rajasekaran, A. Sheth, R. Aggarwal, and K. Sivashanmugam. WSDL-S: Adding Semantics to WSDL - White Paper. Technical report, Large Scale Distributed Information Systems, 2004. http://lsdis.cs.uga.edu/library/download/wsdl-s.pdf.

[18] A. Mocan, E. Cimpian, M. Zaremba, and C. Bussler. Mediation in Web Service Modeling Execution Environment (WSMX). In Information Integration on the Web (IIWeb2004), Toronto, Canada, 2004.

[19] M. Mrissa, C. Ghedira, D. Benslimane, and Z. Maamar. A context model for semantic mediation in web services composition. In D. W. Embley, A. Olivé, and S. Ram, editors, ER, volume 4215 of Lecture Notes in Computer Science, pages 12-25. Springer, 2006.

[20] M. Mrissa, C. Ghedira, D. Benslimane, and Z. Maamar. Towards Context-based Mediation for Semantic Web Services Composition. In Proceedings of the 18th Int'l Conf. on Software Engineering and Knowledge Engineering (SEKE'2006), San Francisco, California, July 2006.

[21] N. F. Noy. Semantic integration: a survey of ontology-based approaches. SIGMOD Rec., 33(4):65-70, 2004.

[22] N. F. Noy and C. D. Hafner. The state of the art in ontology design: A survey and comparative review. In AI Magazine, volume 18, pages 53-74, Fall 1997.

[23] N. F. Noy and D. Mcguinness. Ontology development 101: A guide to creating your first ontology. Stanford KSL Technical Report KSL-01-05, 2000.

[24] G. Schreiber and M. Dean. Owl web ontology language reference. http://www.w3.org/TR/2004/REC-owl-ref-20040210/, February 2004.

[25] B. Spencer and S. Liu. Inferring data transformation rules to integrate semantic web services. In S. A. McIlraith, D. Plexousakis, and F. van Harmelen, editors, Int'l Semantic Web Conference, volume 3298 of Lecture Notes in Computer Science, pages 456-470. Springer, 2004.

[26] UDDI. Universal Description, Discovery, and Integration of Business for the Web, Oct. 2001. URL: http://www.uddi.org.

[27] W3C. XML Schema Part 2: Datatypes Second Edition. Technical report, W3C, October 2004. http://www.w3.org/TR/xmlschema-2/.

[28] G. Wiederhold. Mediators in the architecture of future information systems. IEEE Computer, 25(3):38-49, 1992.

[29] A. B. Williams, A. Padmanabhan, and M. B. Blake. Experimentation with local consensus ontologies with implications for automated service composition. IEEE Trans. Knowl. Data Eng., 17(7):969-981, 2005. 